\title{
Preferential attachment in the growth of social networks: The internet encyclopedia Wikipedia
}

\author{
A. Capocci, ${ }^{1}$ V. D. P. Servedio, ${ }^{1,2}$ F. Colaiori, ${ }^{3}$ L. S. Buriol,,${ }^{2,4}$ D. Donato, ${ }^{2}$ S. Leonardi, ${ }^{2}$ and G. Caldarelli ${ }^{1,3}$ \\ ${ }^{1}$ Centro Studi e Ricerche E. Fermi, Compendio Viminale, Roma, Italy \\ ${ }^{2}$ Dipartimento di Informatica e Sistemistica, Università di Roma "La Sapienza," Via Salaria 113, 00198 Roma, Italy \\ ${ }^{3}$ CNR-INFM(SMC) Istituto dei Sistemi Complessi and Dipartimento di Fisica, Università di Roma "La Sapienza," \\ Piazzale Aldo Moro 2, 00185, Roma, Italy \\ ${ }^{4}$ Computer Science Department, Federal University of Rio Grande do Sul, Porto Alegre/RS, Brazil
}

(Received 17 February 2006; revised manuscript received 21 July 2006; published 25 September 2006)

We present an analysis of the statistical properties and growth of the free on-line encyclopedia Wikipedia. By describing topics by vertices and hyperlinks between them as edges, we can represent this encyclopedia as a directed graph. The topological properties of this graph are in close analogy with those of the World Wide Web, despite the very different growth mechanism. In particular, we measure a scale-invariant distribution of the in and out degree and we are able to reproduce these features by means of a simple statistical model. As a major consequence, Wikipedia growth can be described by local rules such as the preferential attachment mechanism, though users, who are responsible of its evolution, can act globally on the network.

DOI: 10.1103/PhysRevE.74.036116

PACS number(s): 89.75.Hc, 89.75.Da, 89.75.Fb

Statistical properties of social networks have become a major research topic in statistical physics of scale-free networks $[1,2]$. Social networks can be of different types but may be classified either belonging to collaboration systems or information systems. Among collaboration systems we can find, e.g., scientific coauthorship networks [3], where edges are drawn between scientists and those who coauthored the same paper; or actors collaboration networks [4], where edges are drawn between actors playing in the same movie. Information systems are the result of human interaction, intended in a broad sense. We mention here the network of sexual contacts [5], where the study of its statistical properties is strictly related to the spreading of infectious diseases [6], and the World Wide Web (WWW), which is often put outside the social networks category due to its peculiarities [7].

In this paper, we analyze the graph of Wikipedia [8], a virtual on-line encyclopedia. This topic attracted very much interest in recent times $[9,10]$ because of its topological structure. Wikipedia grows constantly as new entries are continuously added by users through the Internet. Thanks to the Wiki software $[11,12]$, any user can introduce new entries and modify the existing ones. It is natural to represent this system as a directed graph, where vertices correspond to entries and edges to hyperlinks, the latter autonomously drawn between various entries by independent contributors.

We find that the Wikipedia graph exhibits a topological bow-tie-like structure, as does the WWW [13]. Moreover, the frequency distributions of the number of incoming (indegree) and outgoing (out-degree) edges show fat-tail powerlaw behaviors. Further, the in degrees of connected vertices are not correlated. These last two findings suggest that edges are not drawn toward and from existing topics uniformly. Rather, the large number of incoming and outgoing edges of a node increases the probability of acquiring new incoming and outgoing edges, respectively. In the literature concerning scale-free networks, this phenomenon is called "preferential attachment" [4] and is explained in detail below.

Wikipedia is an intriguing research object from a sociologist's point of view: pages are published by a number of independent individuals in various languages, covering topics they consider relevant and about which they believe to be competent. Our dataset encompasses the whole history of the Wikipedia database, reporting any addition or modification to the encyclopedia. Therefore, the rather broad information contained in the Wikipedia dataset can be used to validate existing models for the development of scale-free networks. In particular, we found here one of the first large-scale confirmations of the preferential attachment, or "rich-get-richer," rule. This result is rather surprising, since preferential attachment is usually associated to network growth mechanisms triggered by local events: in the WWW, for instance, webmasters have control on their own web pages and outgoing hyperlinks, and cannot modify the rest of the network by adding edges elsewhere. Instead, by the "Wiki" technology a single user can edit an unlimited number of topics and add edges within the Wikipedia network.

The dataset of Wikipedia at the time of submission of this analysis is made of pages in about 100 different languages; the largest subset is almost 1000000 pages of the English version. All sets are still growing at an exponential pace [14]. The dataset we considered in our analysis (June 2004) are smaller and correspond to the figures given in Table I with the largest dataset (English) of about 300000 vertices.

A detailed analysis of the algorithms used to crawl such data is presented elsewhere [15]. Here, we start our analysis

TABLE I. Size of the graph as collected in the dump of June 13, 2004.

\begin{tabular}{ccc}
\hline \hline Language & Vertices & Edges \\
\hline Portuguese & 8645 & 51231 \\
Italian & 13132 & 159965 \\
Spanish & 27262 & 288766 \\
French & 42987 & 660401 \\
German & 116251 & 2163405 \\
English & 339834 & 5278037 \\
\hline \hline
\end{tabular}




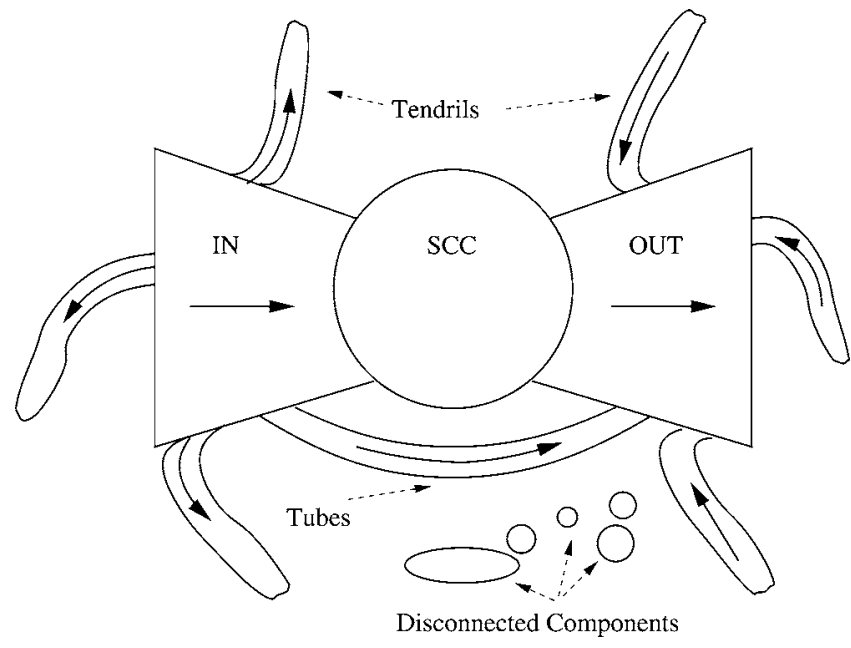

FIG. 1. The shape of the Wikipedia network.

by considering a typical taxonomy of regions introduced for the WWW [13]. The first region includes pages that are mutually reachable by traveling on the graph, named the strongly connected component (SCC) in graph theory; pages from which one reaches the SCC form the second region, the IN component, while the OUT component encompasses the pages reached from the SCC. A fourth region, named TENDRILS, gathers pages reachable from the IN component and pointing neither to the SCC nor the OUT region. TENDRILS also includes those pages that point to the OUT region but do not belong to any of the other defined regions. Finally TUBES connect directly IN and OUT regions, and few pages are totally disconnected (DISC). The result is the so-called bow-tie structure shown in Fig. 1. As a general remark, Wikipedia shows a rather large interconnection since most of the vertices are in the SCC (Table II).

A general dynamical process of network formation allows the deletion and rewiring of existing edges. Unfortunately we do not have a direct estimation of the occurrence of the deletion process. Deleting an edge in the graph of Wikipedia means breaking a logical connection between two topics. It is to our opinion safe to assume that the user who first added that edge did it on purpose and not by mistake. Would it be a mistake, the cooperative system of Wikipedia would remove it very soon so that it would be very hard to detect it in two "snapshots" of Wikipedia, that is, the Wikipedia graph observed at different times.

To be sure of that, we monitored the presence of links between a snapshot and the following one, taken once per week. We found that all edges in a snapshot occur in the following one too, thus confirming indirectly that the edge deletion process is a rare, fast event.

Analogously to the deletion process, we expect that the pure edge rewiring would be rather small. Pure edge rewiring means to move a tip from one existing topic to another, which happens in case of mistakes too. Most of the rewiring includes changes in topic names violating the standard requirements of Wikipedia, e.g., History_of_USA instead of the correct form HistoryOfUSA. In the latter example, Wikipedia created a new topic with the wrong name. This is not considered as a striking error by the Wikipedia community and users let that edge through the system, until an administrator checks them out and renames the wrong topics. Although we quantified this process to occur almost $10 \%$ of times in the English Wikipedia, it will not affect the validity of our model we introduce in the following.

The key quantities characterizing the structure of an oriented network are the in-degree $\left(k_{\text {in }}\right)$ and out-degree $\left(k_{\text {out }}\right)$ distributions. As shown in Fig. 2, both distributions display an algebraic decay, of the kind $P\left(k_{\text {in,out }}\right) \propto k_{\text {in,out }}^{-\gamma_{\text {in, }} \text {, with } 2}$ $\leqslant \gamma^{\text {in,out }} \leqslant 2.2$. Actually, in the case of the out-degree distribution, the value of the exponent seems to depend upon the size of the system as well as the region chosen for the fit. Given the sharp cutoff in this distribution, the cumulative method of plotting in this case could result in a larger value of the exponent.

We proceeded further by studying the dynamics of the network growth. The analysis has been made in order to validate the current paradigm explaining the formation of scale-free networks, introduced by the Barabási-Albert (BA) model [1]. The latter is based on the interplay of two ingredients: growth and preferential attachment. In the BA model, new vertices are added to the graph at discrete time steps and a fixed number $m$ of edges connects each new vertex to the old ones. The preferential attachment rule corresponds to assigning a probability $\Pi\left(k_{i}\right) \sim k_{i}$ that a new vertex is connected to an existing vertex $i$ whose degree is $k_{i}$. This elementary process, already introduced in 1955 by Simon in another context [16], generates a nonoriented network where the degree follows a power-law distribution.

To observe such a mechanism in a real network, one builds the histogram of the degree of the vertices acquiring new connections at each time $t$, weighted by the inverse probability $[P(k, t)]^{-1}=N(t) / n(k, t)$, where $N(t)$ is the number of vertices at time $t$ and $n(k, t)$ is the number of vertices

TABLE II. Size of the bow-tie components of the Wikipedia for various languages. Each entry in the table presents the percentage of vertices of the corresponding graph that belong to the indicated bow-tie component.

\begin{tabular}{ccccccc}
\hline \hline DB & SCC & IN & OUT & TENDRILS & TUBE S & DISC \\
\hline PT & 67.14 & 6.79 & 15.85 & 1.65 & 0.03 & 7.50 \\
IT & 82.76 & 6.83 & 6.81 & 0.52 & 0.00 & 3.10 \\
ES & 71.86 & 12.01 & 8.15 & 2.76 & 0.07 & 6.34 \\
FR & 82.57 & 6.12 & 7.89 & 0.38 & 0.00 & 3.04 \\
DE & 89.05 & 5.61 & 3.95 & 0.10 & 0.00 & 1.29 \\
EN & 82.41 & 6.63 & 6.73 & 0.57 & 0.02 & 3.65 \\
\hline \hline
\end{tabular}




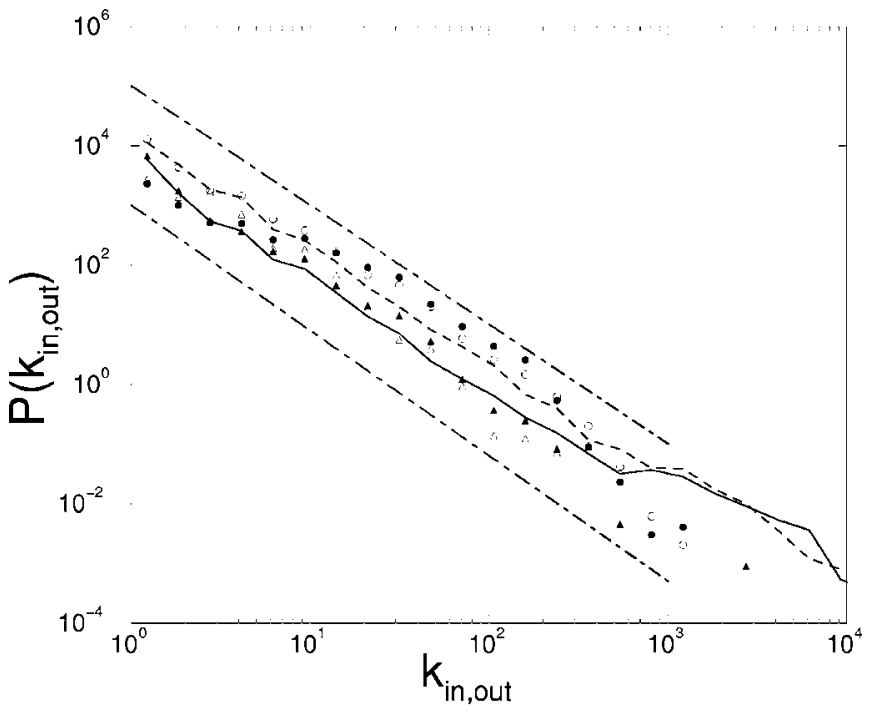

FIG. 2. In-degree (white symbols) and out-degree (filled symbols) distributions for the Wikipedia English (circles) and Portuguese (triangles) graph. The solid line and the dashed line represent simulation results for the in-degree and the out-degree respectively, for a number of 10 edges added to the network per time step. Dotted-dashed lines show the $k_{\text {in,out }}^{-2.1}$ (bottom line) and the $k_{\text {in,out }}^{-2}$ (top line) behavior, as a guide for the eye.

with in-degree $k$ at time $t$ [17]. The weighting factor $1 / P(k, t)$ is necessary to take into account the multiplicity of nodes with the same degree. In fact, the conditional probability that a node $i$ with degree $k_{i}$ acquires a new node at time $i$, is proportional to $\Pi\left(k_{i}\right) / P\left(k_{i}, t\right)$.

Since the Wikipedia network is oriented, the preferential attachment must be verified in both directions. In particular, we have observed how the probability of acquiring a new incoming (outgoing) edge depends on the present in (out) degree of a vertex. The result for the main Wikipedia network (the English one) is reported in Fig. 3.

With linear preferential attachment, as supposed by the BA model, both plots should be linear over the entire range of degree; here we recover this behavior only partly. This is not surprising, since several measurements reported in literature display strong deviations from a linear behavior [18] for large values of the degree, even in networks with an inherent preferential attachment. Such behavior is the result of finite size effects [17]. By carefully observing Fig. 3 we note that for certain datasets (e.g., English), the slope of the growth of $\Pi$ at small connectivities might follow a sublinear law, thus not completely ruling out different mechanisms more complicated than preferential attachment. This is an important issue, since a sublinear preferential attachment fails to produce scale-free networks [19]. However, since the sublinear exponent is very close to unity (ca. 0.9 for the English Wikipedia), we shall consider it as strictly unity in order to define a simplified model able to reproduce the stylized facts of the systems.

Further, it is worth to mention that the preferential attachment in Wikipedia has a somewhat different nature than in other networks. Here, most of the times, edges are added between already existing vertices, unlike the BA model.

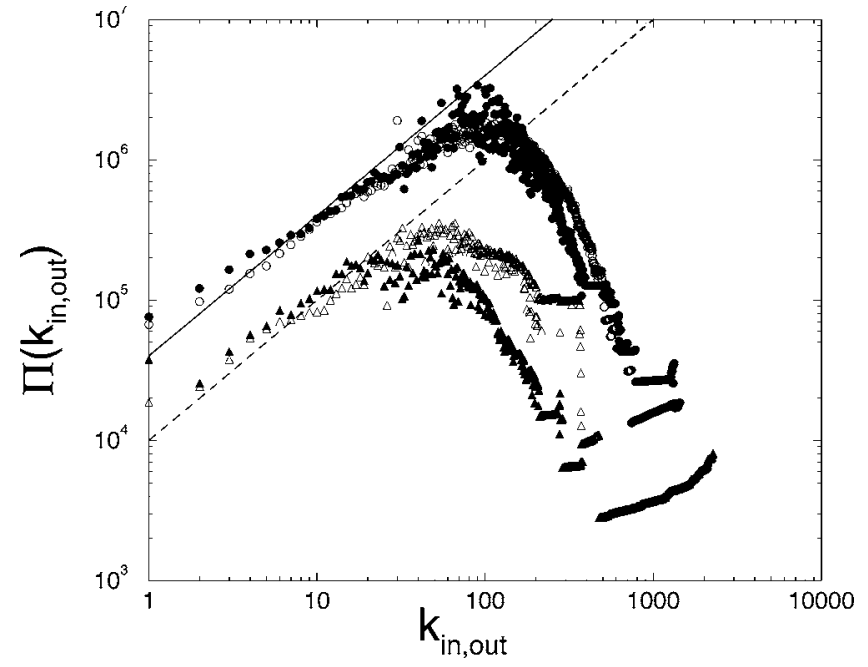

FIG. 3. The preferential attachment for the in degree and the out degree in the English and Portuguese Wikipedia network. The solid line represents the linear preferential attachment hypothesis $\Pi$ $\sim k_{\text {in,out }}$.

To draw a more complete picture of the Wikipedia network, we have also measured the correlations between the in and out degrees of connected pages. The relevance of this quantity is emphasized by several examples of complex networks shown to be fully characterized by their degree distribution and degree-degree correlations [20]. Among other quantities, suitable measure of such correlations is represented by the average degree $K^{(n n)}(k)$ of vertices connected to vertices with degree $k$ (for simplicity, here we refer to a nonoriented network to explain the notation) [21]. These quantities are particularly interesting when studying social networks. As for other social networks, the collaborative networks studied so far are characterized by assorted mixing, i.e., edges preferably connect vertices with similar degrees [7]. This picture would reflect in a $K^{(n n)}(k)$ growing with respect to $k$. If $K^{(n n)}(k)$ (decays) grows with $k$, vertices with similar degrees are (un)likely to be connected. This appears to be a clear cutting method to establish whether a complex network belongs to the realm of social networks, if other considerations turn ambiguous [22].

In the case of an oriented network, such as Wikipedia, one has many options while performing such assessment, since one could measure the correlations between the in or the out degrees of neighbor vertices, along incoming or outgoing edges. We chose to study the average in-degree $K_{\text {in }}^{(n n)}\left(k_{\text {in }}\right)$ of upstream neighbors, i.e., pointing to vertices with in-degree $k_{\text {in. }}$ By focusing on the in-degree and on the incoming edges, we expect to extract information about the collective behavior of Wikipedia contributors and filter out their individual peculiarities: the latter have a strong impact on the outdegree of a vertex and on the choice of its outgoing edges, since contributors often focus on a single Wikipedia topic [14].

Our analysis shows a substantial lack of correlation between the in degrees of a vertex and the average in-degree of its upstream neighboring vertices. So, as reported in Fig. 4, incoming edges carry no information about the in degrees of 


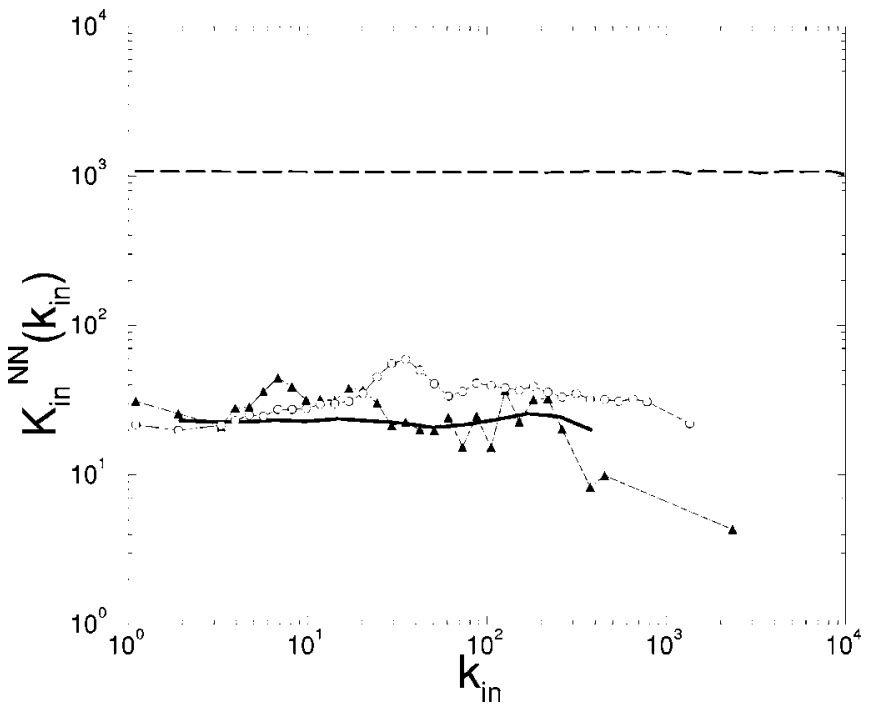

FIG. 4. The average observed neighbors' in degree, computed along incoming edges, as a function of the in degree in the case of the English (circles) and Portuguese (triangles) Wikipedia, compared to the simulations of the model with $N=20000, M=10, R_{1}$ $=0.026$ and $R_{2}=0.091$ (dashed line above), averaged over 100 realizations. A version of the same model where the first $0.5 \%$ of vertices have been removed to reduce the initial condition impact, is also shown as the thick solid line through the observed data.

the connected vertices, since $K^{(n n)}\left(k_{\text {in }}\right)$ display no clear increasing or decreasing behavior when plotted against $k_{\text {in }}$.

A more complete picture of the correlation between vertices can be given by considering the communities formed by similar vertices. In what follows we assume that communities are characterized by cluster of vertices exchanging reciprocal links with a density larger than the average one. In this approach we consider the graph as nonoriented. The study of communities is one of the most interesting aspects in the analysis of scale free networks; unfortunately, it is also one of the most difficult both from a theoretical and a numerical point of view.

Here, we report the results for the Portuguese Wikipedia dataset, small enough to be analyzed by clustering methods which are often computationally demanding. The encyclopedic character of Wikipedia leads unequivocally to the formation of groups of nodes with high link density (communities), reflecting the thematic division of human knowledge. This characteristic highly grouped structure is a typical feature of social networks and sometimes it can be indirectly revealed by the assorted mixing character of networks [22]. As already pointed out, the determination of the community structure of a network is generally not a simple task so that practically one is satisfied by an approximate view of it. To this purpose many different algorithms were proposed in the literature [23]. One possible method involves the study of the eigenvalues and eigenvectors of the normal matrix of a graph $[24,25]$. The idea behind this method is that a graph whose structure is characterize by the presence of strong communities results in a normal matrix made mostly of blocks (and therefore with particular eigenvalue spectrum).

In Fig. 5 we show the value of the first 100 largest eigen-

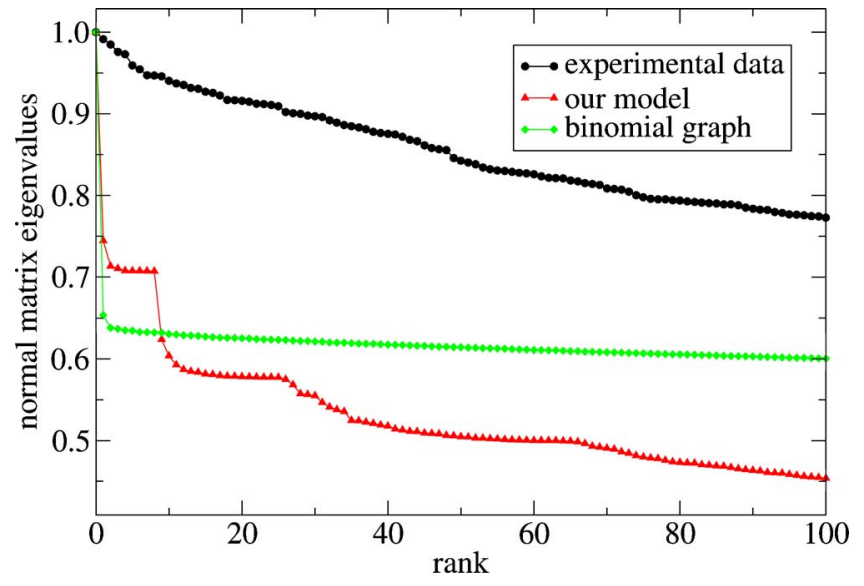

FIG. 5. (Color online) First 100 eigenvalues of the normal matrix associated to the measured Portuguese Wikipedia (circles), to the network of the same order generated by our model (triangles), and the binomial network of the same order and average degree (diamonds). The presence of a clear community structure is revealed by the large number of eigenvalues around the unity.

values of the normal matrix associated with the Portuguese Wikipedia graph with all links considered as undirected, together with the eigenvalues of the graph generated by our model and the eigenvalues of a binomial graph with the same average node connectivity. The presence of a community structure in the Portuguese Wikipedia is revealed by the great number of eigenvalues of the associated normal matrix around 1. In particular, the three most clear communities detectable were in the area of Brazilian movies and actors (ca. 100 entries), medical terms (ca. 300 entries), geographical terms (ca. 2000 entries). We did not perform the analysis of the community size probability distribution where we expect a fat-tailed behavior. In fact, it was shown in Ref. [26] that even in the case of a binomial graph one obtains a power-law distribution of community sizes as an artifact of the community detection algorithms.

The power-law distribution of the connectivities and the absence of degree-degree correlations can be modeled by a simple application of the preferential attachment principle. Let us consider the following evolution rule, similar to existing models of rewiring already $[27,28]$, for a growing directed network such as Wikipedia: at each time step, a vertex is added to the network, and is connected to the existing vertices by $M$ oriented edges; the direction of each edge is drawn at random: with probability $R_{1}$ the edge leaves the new vertex pointing to an existing one chosen with probability proportional to its in degree; with probability $R_{2}$, the edge points to the new vertex, and the source vertex is chosen with probability proportional to its out degree. Finally, with probability $R_{3}=1-R_{1}-R_{2}$ the edge is added between existing vertices: the source vertex is chosen with probability proportional to the out degree, while the destination vertex is chosen with probability proportional to the in degree.

By solving the rate equations for $k_{\text {in }}$ and $k_{\text {out }}$ by standard arguments [1], we can show that this mechanism generates power-law distributions of both the in degree and the out degree, $k_{\text {in }}$ and $k_{\text {out }}$,

$$
P\left(k_{\text {in }}\right) \simeq k_{\text {in }}^{-\left[1 /\left(1-R_{2}\right)\right]-1},
$$




$$
P\left(k_{\text {out }}\right) \simeq k_{\text {out }}^{-\left[1 /\left(1-R_{1}\right)\right]-1},
$$

which can be easily verified by numerical simulation. By adopting the values empirically found in the English Wikipedia $R_{1}=0.026, R_{2}=0.091$, and $R_{3}=0.883$, one recovers the same power-law degree distributions of the real network, as shown in Fig. 2.

As regards the English version of Wikipedia a largely dominant fraction $R_{1}$ of new edges is created between two existing pages, while smaller fractions $R_{2}$ and $R_{3}$ of edges, respectively, point or leave a newly added vertex. By analyzing the update record of the above English Wikipedia database we measured that, in the period 2001-2004, such fractions took the values $R_{1}=0.883 \pm 0.001, R_{2}=0.026 \pm 0.001$, and $R_{3}=0.091 \pm 0.001$.

The degree-degree correlations $K_{\text {in }}^{(n n)}\left(k_{\text {in }}\right)$ can be computed analytically by the same lines of reasoning described in Refs. $[22,29]$, and for $1 \ll k_{\text {in }} \ll N$ we have

$$
K_{\text {in }}^{(n n)}\left(k_{\text {in }}\right) \sim \frac{M R_{1} R_{2}}{R_{3}} N^{1-R_{1}}
$$

for $R_{3} \neq 0$, the proportionality coefficient depending only on the initial condition of the network, and

$$
K_{\text {in }}^{(n n)}\left(k_{\text {in }}\right) \simeq M R_{1} R_{2} \ln N
$$

for $R_{3}=0$, where $N$ is the network size. Both equations are independent from $k_{\text {in }}$, as confirmed by the simulation reported in Fig. 4 for the same values of $R_{1}, R_{2}$, and $R_{3}$. Therefore, the theoretical degree-degree correlation reproduces qualitatively the observed behavior; to obtain a more accurate quantitative agreement with data, it is sufficient to tune the initial conditions appropriately. As shown in Fig. 4, this can be done by neglecting a small fraction of initial vertices in the network model. In the first case the values of the exponents can vary from -2 (when $R_{1}, R_{2}$, respectively, are 0 ) to minus infinite (when $R_{1}, R_{2}$, respectively, are -1 ). For the expected (empirical) range of variation (i.e., from $2 \%$ to $20 \%) \quad R_{2}=0.02 \rightarrow \gamma_{\text {in }}=2.02, \quad R_{2}=0.2 \rightarrow \gamma_{\text {in }}=2.25, \quad R_{1}=0.02$ $\rightarrow \gamma_{\text {out }}=2.02, R_{1}=0.2 \rightarrow \gamma_{\text {out }}=2.25$. As regards the slope of the average neighbor coefficient, this does not depend upon the values of the parameter. We confirm these behaviors by means of numerical simulations.

Nevertheless, this model does not reproduce the observed complex community structure of Wikipedia. This is some- what reasonable and expected, by considering the strong assumptions made in the model definition. A community structure could arise in models where thematic divisions are taken into account by means of fitness hidden variables [30-32].

In conclusion, the bow-tie structure already observed in the World Wide Web, and the algebraic decay of the indegree and out-degree distribution are observed in the Wikipedia datasets surveyed here. At a deeper level, the structure of the degree-degree correlation also resembles that of a network developed by a simple preferential attachment rule. This has been verified by comparing the Wikipedia dataset to models displaying no correlation between neighbors degrees.

Thus, empirical and theoretical evidences show that traditional models introduced to explain nontrivial features of complex networks by simple algorithms remain qualitatively valid for Wikipedia, whose technological framework would allow a wider variety of evolutionary patterns. This reflects on the role played by the preferential attachment in generating complex networks: such mechanism is traditionally believed to hold when the dissemination of information throughout a social network is not efficient and a "bounded rationality" hypothesis $[33,34]$ is assumed. In the WWW, for example, the preferential attachment is the result of the difficulty for a webmaster to identify optimal sources of information to refer to, favoring the herding behavior which generates the "rich-get-richer" rule. One would expect the coordination of the collaborative effort to be more effective in the Wikipedia environment since any authoritative agent can use his expertise to tune the linkage from and toward any page in order to optimize information mining. Nevertheless, empirical evidences show that the statistical properties of Wikipedia do not differ substantially from those of the WWW. This suggests two possible scenarios: preferential attachment may be the consequence of the intrinsic organization of the underlying knowledge; alternatively, the preferential attachment mechanism emerges because the Wiki technical capabilities are not fully exploited by Wikipedia contributors: if this is the case, their focus on each specific subject puts much more effort in building a single Wiki entry, with little attention toward the global efficiency of the organization of information across the whole encyclopedia.

The authors acknowledge support from the European Project DELIS.
[1] R. Albert and A.-L. Barabási, Rev. Mod. Phys. 74, 47 (2002).

[2] S. N. Dorogovtsev and J. F. F. Mendes, Evolution of Networks: From Biological Nets to the Internet and Www (Oxford University Press, Oxford, 2003).

[3] M. E. J. Newman, Phys. Rev. E 64, 016131 (2001); 64, 016132 (2001).

[4] A.-L. Barabási and R. Albert, Science 286, 509 (1999).

[5] F. Liljeros et al., Nature (London) 411, 907 (2001).

[6] R. Pastor-Satorras and A. Vespignani, Phys. Rev. Lett. 86, 3200 (2001); Phys. Rev. E 65, 035108(R) (2002).
[7] M. E. J. Newman, Phys. Rev. Lett. 89, 208701 (2002).

[8] http://www.wikipedia.org/

[9] T. Holloway, M. Božičević, and K. Börner, e-print cs.IR/ 0512085.

[10] After our submission on the arXiv, a new paper appeared on these topics V. Zlatić, M. Božičević, H. Štefančić, and M. Domazet, e-print physics/0602149.

[11] http://en.wikipedia.org/wiki/Wiki

[12] F. B. Viégas, M. Wattenberg, and K. Dave, CHI '04: Proceedings of the SIGCHI conference on Human factors in comput- 
ing systems, 2004, p. 575.

[13] A. Z. Broder et al., Comput. Netw. 33, 309 (2000).

[14] J. Voss, Proceedings 10th International Conference of the International Society for Scientometrics and Informetrics 2005.

[15] D. Donato et al., A library of software tools for performing measures on large networks, COSIN Tech-report Deliverable D13 (http://www.dis.uniroma1.it/ cosin/publications), 2004.

[16] H. A. Simon, Biometrika 42, 425 (1955).

[17] M. E. J. Newman, Phys. Rev. E 64, 025102(R) (2001).

[18] M. Peltomäki and M. Alava, e-print physics/0508027.

[19] P. L. Krapivsky, S. Redner, and F. Leyvraz, Phys. Rev. Lett. 85, 4629 (2000).

[20] G. Bianconi, G. Caldarelli, and A. Capocci, Phys. Rev. E 71, 066116 (2005).

[21] A. Vázquez, R. Pastor-Satorras, and A. Vespignani, Phys. Rev. E 65, 066130 (2002); S. Maslov and K. Sneppen, Science 296, 910 (2002).

[22] A. Capocci and F. Colaiori, e-print cond-mat/0506509.

[23] M. E. J. Newman, Eur. Phys. J. B 38, 321 (2004).
[24] A. Capocci et al., Physica A 352, 669 (2005).

[25] M. E. J. Newman, e-print physics/0605087.

[26] G. Caldarelli, C. C. Cartozo, P. De Los Rios, and V. D. P. Servedio, Phys. Rev. E 69, 035101(R) (2004).

[27] P. L. Krapivsky, G. J. Rodgers, and S. Redner, Phys. Rev. Lett. 86, 5401 (2001).

[28] B. Tadic, Physica A 293, 273 (2001).

[29] A. Barrat and R. Pastor-Satorras, Phys. Rev. E 71, 036127 (2005).

[30] G. Caldarelli, G. Capocci, P. De Los Rios, and M. A. Munoz, Phys. Rev. Lett. 89, 258702 (2002).

[31] D.-H. Kim, B. Kahnga, and D. Kim, Eur. Phys. J. B 38, 305 (2004).

[32] M. Boguñá, R. Pastor-Satorras, A. Diaz-Guilera, and A. Arenas, Phys. Rev. E 70, 056122 (2004).

[33] M. Rosvall and K. Sneppen, Phys. Rev. Lett. 91, 178701 (2003).

[34] S. Mossa, M. Barthelemy, H. E. Stanley, and L. A. Nunes Amaral, Phys. Rev. Lett. 88, 138701 (2002). 